\title{
A novel approach to the evaluation of contact thermal resistance at soil-structure interfaces
}

\author{
João Diogo Figueira ${ }^{1, *}$, Peter Bourne-Webb ${ }^{1}$, and Teresa Bodas Freitas ${ }^{1}$ \\ 1 CERIS, Instituto Superior Técnico, Universidade de Lisboa, Lisboa, Portugal
}

\begin{abstract}
Considering the need to reduce our dependence on unsustainable energy sources and reducing the carbon footprint associated with building climate control, shallow geothermal energy represents an attractive sustainable technology for providing renewable heating and cooling. The temperature field generated around ground-coupled heat exchangers, and thus their energy efficiency, fundamentally depends on the heat transfer mechanism and the thermal properties of the materials involved. While the thermal properties of materials that make up the system can be defined with some certainty, little is known about the impact of contact thermal resistance at the soil-structure interface. Contact thermal resistance will reduce heat exchange efficiency and increase mechanical impacts associated with temperature changes within energy geo-structures. This paper describes a laboratory test method to quantify the contact thermal resistance of soil-concrete interfaces. The methodology is first evaluated using numerical analysis, and then validated against a test using a limestone aggregate concrete and fine, silica sand at differing levels of compaction.
\end{abstract}

\section{Introduction}

Shallow geothermal energy (SGE) has the potential to successfully provide renewable heating and cooling to buildings through heat exchange with the ground they stand on. This heat exchange is achieved through underground heat exchanger elements with imbedded pipes (closed-loop system). The heat transfer phenomena is dominated by conduction processes, but convection can also contribute if the groundwater is mobile. Contact thermal resistance (CTR) disturbs the conductive heat flow between two materials in contact with each other. CTR occurs due to geometric irregularities, surface micro-hardness \& cleanliness, contact pressure and void filling materials [1]. In SGE systems, there are contacts between differing materials associated with the heat exchange process (e.g. soil, rock, grout and concrete, and pipes). As these materials have thermal resistance values between those of insulators and metals, it is not apparent whether the CTR effect will be significant in SGE systems or not.

In the design of borehole heat exchangers, lumped resistances, determined from field tests, are used. Such models include the thermal resistance of all materials and contacts within the heat exchanger system [2]. When it comes to SGE systems using geo-structures as the heat exchanger units (i.e. energy piles, walls and tunnels), it is not clear that the lumped resistance approach can always be quantified and used.

The focus of this work is the CTR at the soil-structure interface, henceforth referred to as the geo-CTR. The only attempt to quantify geo-CTR found is in [3], while
$[4,5]$ examine the effect of CTR in terms of ill-fitting borehole liners. When it comes to numerical studies of heat flow between structures and the ground, the vast majority of works do not even refer to geo-CTR. Finite values for geo-CTR were used by $[6,7]$ but without any justification for the values used. In the back-analysis of energy pile thermal tests $[8,9]$ have identified that a geoCTR had to be introduced in order to reproduce the tests numerically. [10] evaluated the impact of geo-CTR on the heat exchange process between a concrete pile foundation and the ground and concluded that its impact is significant for geo-CTR values larger than 0.04 $\mathrm{m}^{2} \mathrm{~K} / \mathrm{W}$. This compares with the values used by $[8,9]$ which were in the range of 0.17 to $0.35 \mathrm{~m}^{2} \mathrm{~K} / \mathrm{W}$ which suggests that geo-CTR is important for the reliability of thermal and thermo-mechanical design of energy geostructures. In the following, a novel methodology that has been developed in order to estimate geo-CTR is described and demonstrated.

\section{Methodology}

Procedures for evaluating the CTR between metallic or ceramic materials in contact are well established and [11] identify that steady state methods are most suitable for bulk materials. In these methods,

(i) The two materials are set in contact and heat flow across the contact is established;

(ii) The heat flow is assumed to be one-dimensional (no heat loss) and that the heat flow at the contact, $\mathrm{q}$ is the same as the heat flow at the sample ends;

\footnotetext{
* Corresponding author: joao.figueira@tecnico.ulisboa.pt
} 
(iii) Temperatures are measured along the axis of the material samples, either side of the contact;

(iv) Temperatures are extrapolated to either side of the contact (Fig. 1), from which the drop in temperature across the interface, $\Delta \mathrm{T}$ is established;

(v) The CTR is then obtained via (1).

$$
R_{\text {int }}=\Delta T / \mathrm{q}
$$

For testing at the scale needed for soil and concrete materials with medium thermal conductivities, onedimensional heat flow cannot be maintained due to lateral heat losses $[12,13]$. Common methodologies to reduce lateral heat losses, such as performing the test in vacuum, become impractical when using samples with dimensions in the order of decimetres (necessary to obtain a representative sample of the concrete).

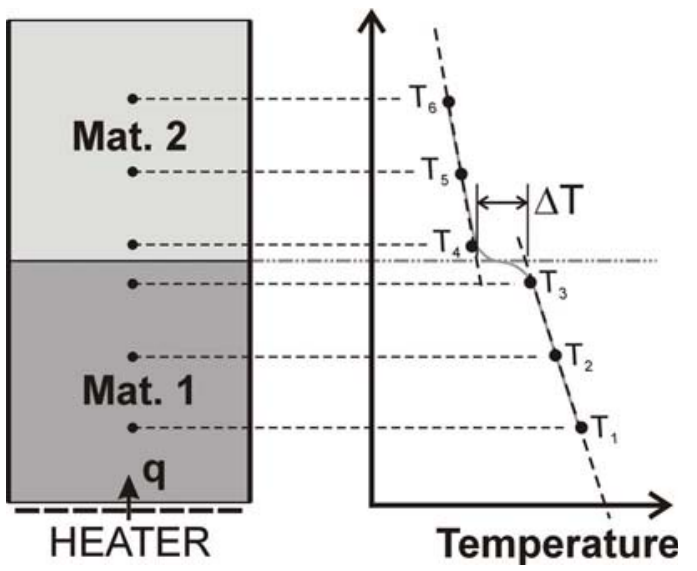

Fig. 1. Schematic layout for CTR determination.

In the proposed test method, two cylindrical samples of each material (e.g. concrete and soil) are formed inside a plastic tube, which itself is contained within a larger enclosure filled with expanded polystyrene chips. For the geo-CTR evaluation, temperature sensors were introduced along the axis of the samples, three in each sample (the first $1.5 \mathrm{~cm}$ from the contact and then at 6 $\mathrm{cm}$ intervals, Fig. 2). Heat was applied at the base of the concrete using a silicon heating pad where the input heat flux, $\mathrm{q}_{1}$, and temperature were recorded. The novelty of the method proposed by the authors lies in the acknowledgement of the significance of the heat losses and the introduction of a thin-film heat flux sensor at the contact to measure directly the heat flux crossing the interface, $\mathrm{q}_{2}$. The value for $\Delta \mathrm{T}$ obtained from the temperature measurements and $\mathrm{q}_{2}$ are then used in (1) to obtain the geo-CTR.

\section{Validation by numerical analysis}

Axisymmetric, transient, thermal finite element analysis of the test set-up was undertaken using the commercial program ABAQUS 2016, with the aim of examining how the test might function, the effect of heat loss and the benefit of the heat flux measurement at the contact.
Analyses were undertaken considering three cases A, B and $\mathrm{C}$ as follows:

The sample geometry modelled was $20 \mathrm{~cm}$ diam. with axial dimensions comprising $20 \mathrm{~cm}$ of concrete and $20 \mathrm{~cm}$ of soil (Fig. 3), which in Case $\mathrm{C}$ were bounded by a $9.6 \mathrm{~mm}$ wall thickness PVC pipe and a further $20 \mathrm{~cm}$ of EPS insulation. Thermal properties for each of the materials are presented in Table 1 . In all cases, a constant temperature of $70^{\circ} \mathrm{C}$ was applied at the base of the concrete.

Case A (one-dimensional heat flow): Mat. 1 Base surface - constant temp. $70^{\circ} \mathrm{C}$, Mat. 2 top surface constant temp. $20^{\circ} \mathrm{C}$, side boundary adiabatic;

Case B (one-dimensional heat flow): Mat. 1 Base surface - constant temp. $70^{\circ} \mathrm{C}$, Mat. 2 top surface - contact conductance of $10 \mathrm{~W} / \mathrm{m}^{2} \mathrm{~K}$ and an air temp. of $20^{\circ} \mathrm{C}$, side boundary adiabatic (zero heat loss);

Case C (two-dimensional heat flow): as Case B with Mat. 2 top surface applied to all top and side boundaries, and internal boundaries with contact conductance values of $25 \mathrm{~W} / \mathrm{m}^{2} \mathrm{~K}$ on all internal contacts except the PVCEPS contact where $1000 \mathrm{~W} / \mathrm{m}^{2} \mathrm{~K}$ was used.

Table 1. Thermal properties used in numerical analysis.

\begin{tabular}{|c|c|c|c|}
\hline Material & $\lambda(\mathbf{W} / \mathbf{m . K})$ & $\rho\left(\mathbf{k g} / \mathbf{m}^{\mathbf{3}}\right)$ & $\mathbf{c}(\mathbf{J} / \mathbf{k g . K})$ \\
\hline Mat. 1 - concrete & 2.0 & 2450 & 940 \\
\hline Mat. 2- soil & 1.0 & 1600 & 1220 \\
\hline PVC Tube & 0.15 & 1395 & 840 \\
\hline EPS Insulation & 0.039 & 15 & 1500 \\
\hline
\end{tabular}

Each analysis was run for a total of 21 days with time steps of 1 hour. Fig. 2 illustrates how the differing thermal boundary conditions affect the temperature fields within each model.

These analyses showed that the measurement of the heat flow at the contact, reduces the error associated with the geo-CTR estimate to around 1\% [14]. This error already considers the errors associated with the extrapolation of the temperature value in the contact from the temperature measurement in a discrete set of points (where the thermocouples are located); and the error associated with the introduction of a heat flux sensor in the interface. By comparison, in the analyses undertaken, when the effects of lateral heat losses are ignored, the error associated with the estimate of geoCTR is $44 \%$.

The numerical analyses have demonstrated that the proposed test methodology should allow the geo-CTR at soil-concrete interfaces to be reliably estimated. The numerical analysis also identified that steady state heat flow was not required to establish the geo-CTR reliably, it requires a relatively modest test time (less than 1 day) to establish the geo-CTR, though this will vary 
somewhat with the thermal properties of the materials being tested.

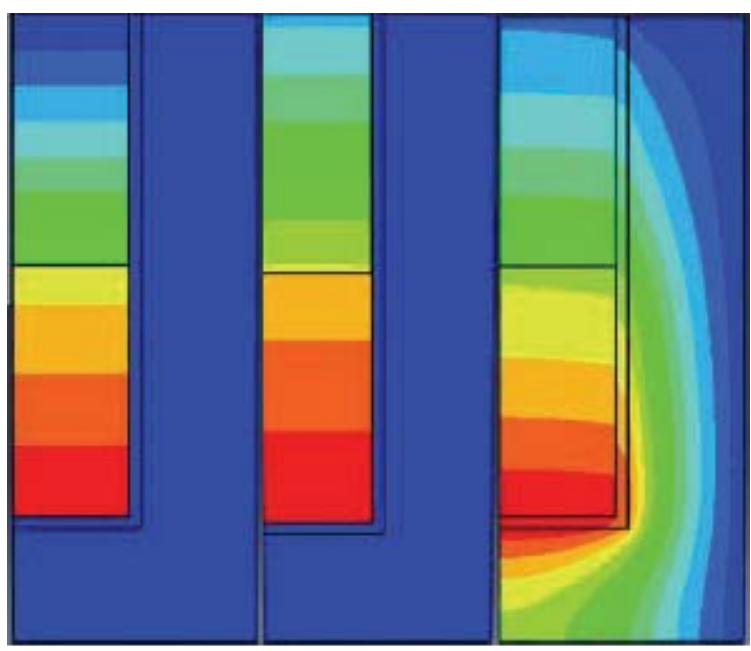

Fig. 2. Temperature fields for Cases A, B and C. Temperatures vary from $20^{\circ} \mathrm{C}$ (in blue) to $70^{\circ} \mathrm{C}$ (in red).

\section{Experimental analysis}

The final test configuration and dimensions are shown in Fig. 3. The final test configuration includes a wooden box clad with $10 \mathrm{~mm}$ plywood which is used to support the tube, and the void around the tube is filled with expanded polystyrene packaging chips in order to provide insulation and to minimise convection within the void (Fig. 4).

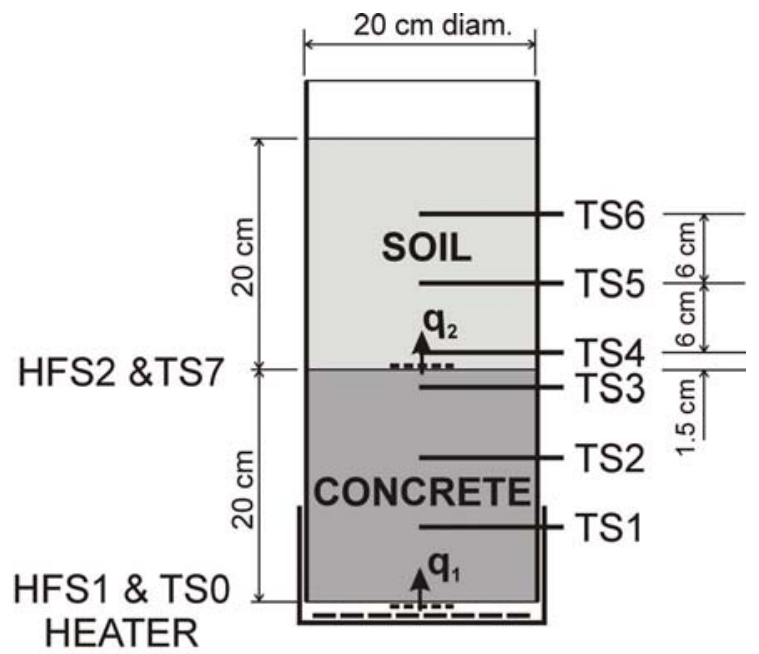
TS: Temperature sensor
HFS: Heat Flow Sensor

Fig. 3. Test dimensions and instrumentation installed.

The concrete used had a 28-day compression strength of $30 \mathrm{MPa}$, a water cement ratio of 0.52 , a ratio of cement to sand to coarse aggregate of 1.0:2.0:2.6, and used limestone aggregates. The concrete was placed carefully in the tube so as not to disturb the ruggedized thermocouples (Type K standard; TS1 - TS3, Fig. 3), and was vibrated to help remove entrained air pockets. The concrete was then allowed to moist cure for around a month. The thermal properties of the concrete sample have not yet been confirmed however BS EN 1992-12:2004 suggests that the thermal conductivity of concrete made with calcareous aggregates should be around 1.30 $\mathrm{W} / \mathrm{m} . \mathrm{K}$.

Before adding the sand sample, a single thin-film heat flow sensor (Omega Engineering HFS-4, 2.062 $\mathrm{mV} /\left(\mathrm{W} / \mathrm{m}^{2}\right)$ sensitivity, discontinued) was placed at the centre of the surface of the concrete where the concretesoil interface would be formed.

The soil infill used in the preliminary testing is a dry, uniform, medium to coarse silica sand with a median particle size, D50, of about $0.6 \mathrm{~mm}$, Fig. 5(a). Minimum and maximum dry density values of 1.34 and $1.58 \mathrm{~g} / \mathrm{m}^{3}$ were obtained, Fig. 5(b). Three needle probe thermal tests on the dry loose sand were performed and the average value for the thermal conductivity of the sand was $0.236 \mathrm{~W} / \mathrm{m} . \mathrm{K}$.

The dry sand was placed as described in the following sections, taking care to ensure that the ruggedized thermocouples (TS4 - TS6) were located correctly along the axis of the soil sample.

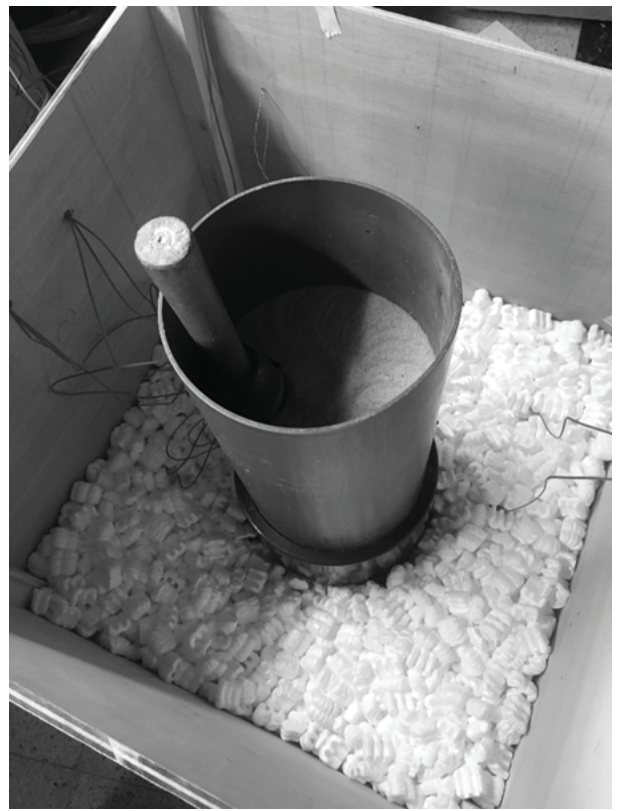

Fig. 4. Illustration of test set-up.

At the base of the column of test materials, Fig. 3, another heat flow sensor was placed between the base of the concrete sample and the heating element $(385 \mathrm{~W}, 18$ $\mathrm{cm}$ diam., silicone rubber heater). The test was run over 2.5 to 3 days with the temperature of the heater being ramped up from room temperature (about $19^{\circ} \mathrm{C}$ ) to the target value of $75^{\circ} \mathrm{C}$ in the first hour. The temperature at the base of the concrete was monitored and maintained to within $\pm 2^{\circ} \mathrm{C}$ using a PID (proportional-integralderivative) process controller. Data from the sensors was captured using an iNET-400 series data acquisition system. 

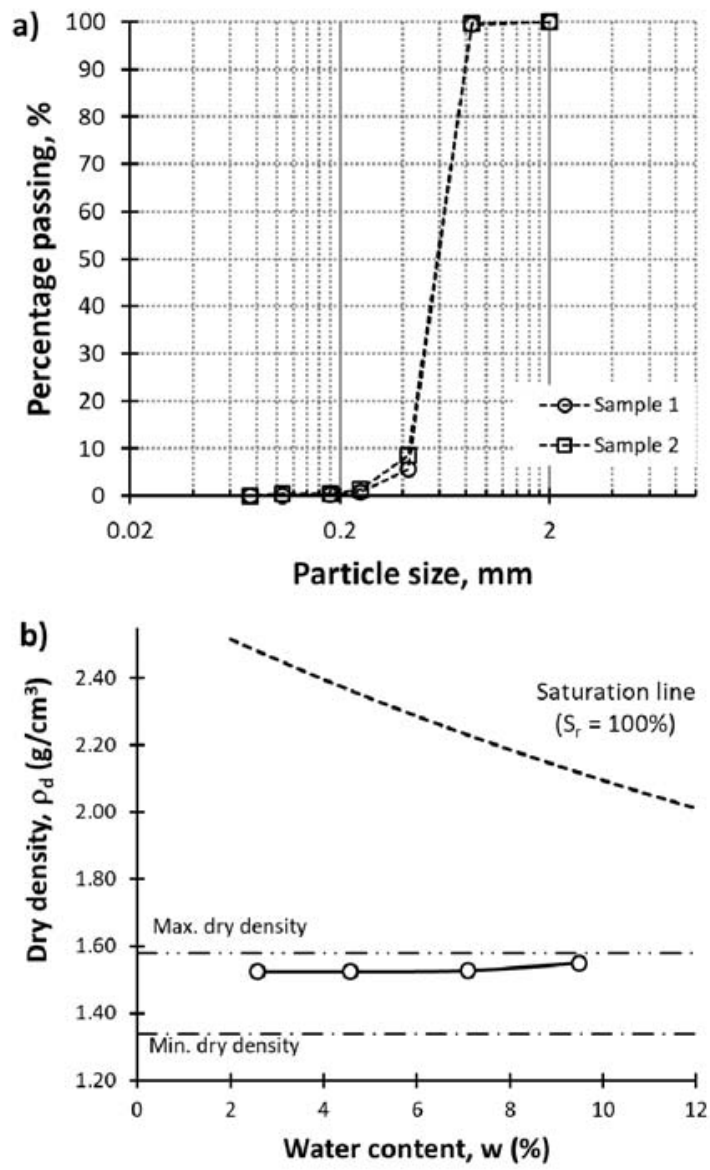

Fig. 5. Sand particle size distribution and compaction curve.

\subsection{Concrete: Loose Sand contact}

Series 1 used sand poured into the tube to form a loose sample and three tests were performed under the same conditions. Fig. 6 illustrates the results from the tests. Fig. 6(a) illustrates the evolution of the temperature along the sample axis, at the temperature sensor locations (filled dots), where it is apparent that after $18 \mathrm{~h}$ of heating, the temperature profile is undistinguishable. The evolution of the temperature drop across the interface is shown in Fig. 6(b), and Fig. 6(c) shows the evolution of the geo-CTR. The average CTR value obtained in this first set of tests is $0.0102 \mathrm{~m}^{2} \mathrm{~K} / \mathrm{W}$ with a standard deviation of $0.00058 \mathrm{~m}^{2} \mathrm{~K} / \mathrm{W}$. The CTR estimation clearly does not present a level of uncertainty as small as the theoretical value suggested by the numerical analysis. However, after 12 hours, the value is stable within $10 \%$ of the value at 60 hours, which is satisfactory.
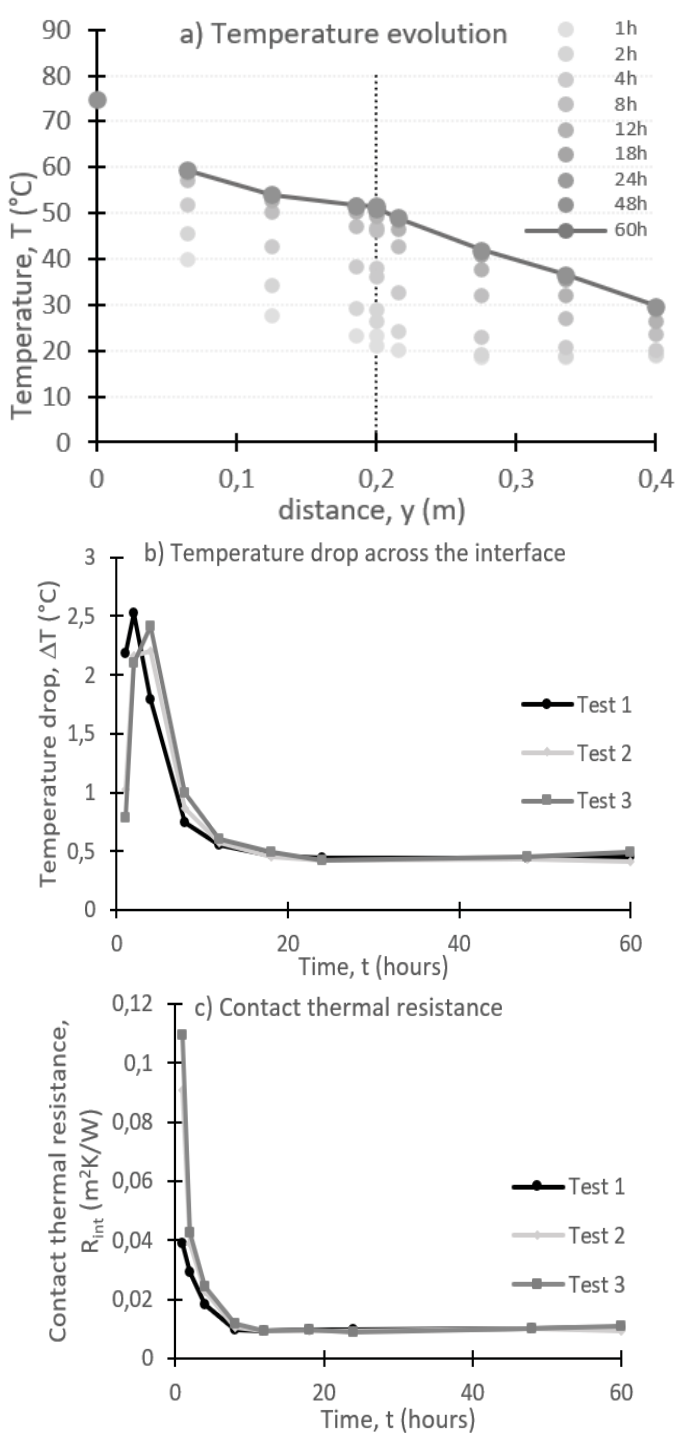

Fig. 6. Concrete: Loose sand geo-CTR test (a) temperature evolution along the sample axis for test 1 , (b) evolution of the temperature drop across the interface, and (c) evolution of the CTR value estimated.

\subsection{Concrete: Dense Sand contact}

Series 2 used a dense sand that was prepared by placing the sand in $2-3 \mathrm{~cm}$ thick layers, to which 20 blows of a $5.58 \mathrm{~kg}$ hammer with a diameter of $100 \mathrm{~mm}$, falling a height of about $5 \mathrm{~cm}$ were applied uniformly across the layer. Again, three tests were performed under the same conditions. Fig. 7 illustrates the results of the three tests undertaken.

Fig. 7 (a) illustrates the evolution of the temperature along the sample axis, at the temperature sensor locations (filled dots), where it is apparent that after $18 \mathrm{~h}$ of heating, the temperature profile is undistinguishable. The evolution of the temperature drop across the interface is shown in Fig. 7(b), and Fig. 7(c) shows the evolution of the CTR estimate. The average CTR value 
obtained in this first set of tests is $0.00074 \mathrm{~m}^{2} \mathrm{~K} / \mathrm{W}$ with a standard deviation of $7.59 \times 10^{-5} \mathrm{~m}^{2} \mathrm{~K} / \mathrm{W}$. The CTR estimation clearly does not present a level of uncertainty as small as the theoretical value suggested by the numerical analysis. When dealing with such a minimal CTR value, the reliability of the measurements gains relevance and the results suggest the need of higher reliability regarding the measuring equipment, as $0.1^{\circ} \mathrm{C}$ precision is $25 \%$ of the $\Delta \mathrm{T}$ in Series 1 tests and $4 \mathrm{x}$ larger than the $\Delta \mathrm{T}$ recorded in the Series 2 tests.
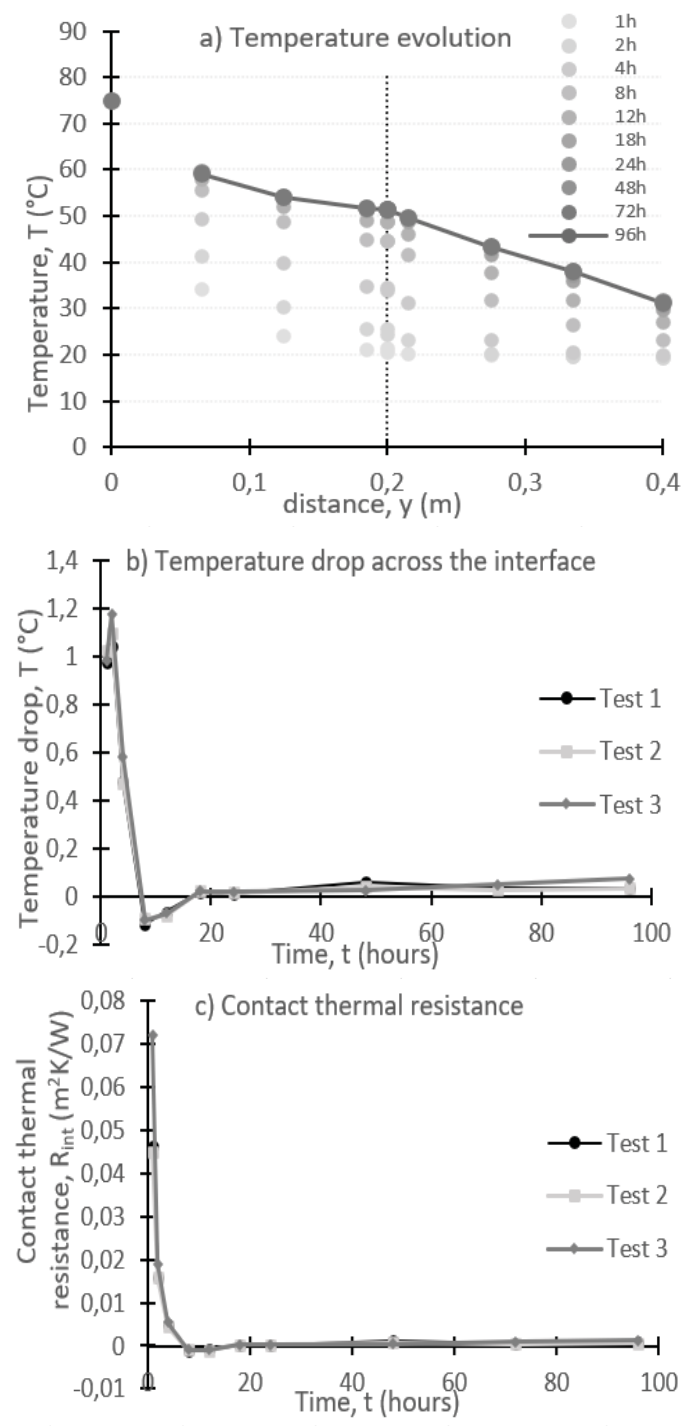

Fig. 7. Concrete: Dense sand geo-CTR test a) temperature evolution along the sample axis for test $1, b$ ) evolution of the temperature drop across the interface, and c) evolution of the CTR value estimated.

\section{Final considerations}

SGE is becoming increasingly attractive as a renewable source of heating and cooling due to the necessity of decarbonizing society. These energy systems depend on the efficiency of the heat transfer mechanisms between the heat exchanger units and the surrounding soil.
These heat transfer mechanisms depend on the thermal properties of the materials involved and on the interface(s) between them. Very little appears to have been done to understand heat flow behaviour across geocontacts, i.e. the contact between the heat exchanger unit and the soil.

A novel laboratory method has been proposed to evaluate the contact thermal resistance between concrete and soil (geo-CTR). This method recognizes that lateral heat losses are inevitable and demonstrates numerically that a better evaluation of the geo-CTR can be made if the heat flow is measured directly on the interface.

A set of laboratory tests evaluating the contact thermal resistance between a concrete and a dry, medium sand at two different initial densities have been presented. The results show that the contact thermal resistance is sensitive to the density of the soil but its value is lower than the values suggested in other studies, e.g. [8, 9] which were obtained for stiff clay-pile interfaces. This suggests that in dry sand, the impact of contact thermal resistance on energy geostructure operation and behaviour would be small.

By better understanding the impact of geo-CTR on heat exchange with the ground, it is expected that more reliable predictions of thermal performance and thermomechanical interactions will be obtained, improving the efficiency and reducing the risks associated with the use of energy geo-structures in the future.

This work was initially developed within the scope of the research grant UID/ECI/04625/2013 financed by CERIS (Civil Engineering Research and Innovation for Sustainability), and continued under the $\mathrm{PhD}$ program of the first author $(\mathrm{SFRH} / \mathrm{BD} / 128845 / 2017)$ and the DEEPCOOL research project (PTDC/ECI-EGC/29083/2017) both funded by the Foundation for Science and Technology (FCT). The authors are grateful for the financial support of the two institutions.

\section{References}

1. M. Yovanovich. Thermal Interface (Joint) Conductance and Resistance. Ther. Int. Cond. and Res.

http://mhtlab.uwaterloo.ca/courses_old/ece309/notes /conduction/cont.pdf (1999)

2. R.A. Beier, M.D. Smith. Borehole thermal resistance from line-source model of in-situ tests. ASHRAE Trans., 108(2), pp. 212-219 (2002).

3. O.J. Svec, L.E. Goodrich, J.H.L. Palmer. Heat transfer characteristics of in-ground heat exchangers. En. Res. 7, pp. 265-278 (1983).

4. L. Wang, H. Liu, Z. Pang, X. Lv. Overall heat transfer coefficient with considering thermal contact resistance in thermal recovery wells. Int. J. of Heat and Mass Transfer 103, pp. 486-500 (2016).

5. G. Hellström, Ground Heat Storage: Thermal analysis of duct storage systems ( $\mathrm{PhD}$ thesis, Lund University, 1991).

6. H.R. Thomas, S.W. Rees. The thermal performance of ground floor slabs - a full scale in situ experiment. Buil. and Env. 34, pp. 139-164 (1999). 
7. A.A. Al-Temeemi, D.J. Harris. The effect of earthcontact on heat transfer through a wall in Kuwait. En. \& Buil. 35, pp. 399-404 (2003)

8. He Qi, Thermal Performance of the Energy Geotechnical Structures (University of Cambridge, 2015).

9. F. Cecinato, R. Piglialepre, F. Loveridge, D. Nicholson, Numerical analysis of thermal cycling during a multi-stage energy pile thermal response test. Proc. 1st Intl. Conf. on Energy Geotechnics, Kiel, Germany, pp. 593-600 (2016).

10. R.M. Freitas Assunção, Thermal and thermalmechanical analysis of thermo-active pile foundations. (IST, University of Lisbon, 2014)

11. Xian Y., Zhang P., Zhai S., Yuan P., Yang D. Experimental characterization methods for thermal contact resistance: A review. Applied Thermal Engineering 130, pp.1530-1548 (2018).

12. C.V. Madhusudana. Accuracy in thermal contact conductance experiments - the effect of heat losses to the surroundings. Int. Comm. Heat Mass Transfer 27, no. 6, pp- 877-891 (2003).

13. J. Low, F.A. Loveridge, W. Powrie. Error analysis of the thermal cell for soil thermal conductivity measurement. Proc. of the Inst. of Civ. Eng. - Geot. Eng. 170, pp. 191-200 (2017).

14. P.J. Bourne-Webb, J.D. de Sousa Figueira, T.M. Bodas Freitas. On the resistance to heat flow across soil-structure interfaces. Energy \& Buildings (2020). (to be published)

15. BS EN 1992-1-2:2004: Design of concrete structures - Part 1-2: General rules - Structural fire design. European Standard. 